
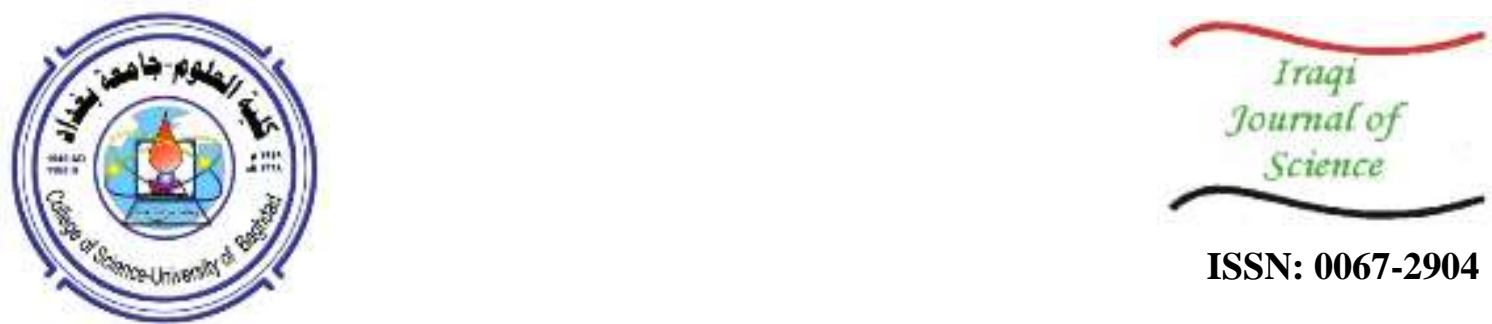

ISSN: 0067-2904

\title{
Intelligent TRIPLE DES with N Round Based on Genetic Algorithm
}

\author{
Mazin Haithem *1, Rana Abdul Rahman Lateef ${ }^{2}$ \\ ${ }^{1}$ Department of Financial and Banking, Baghdad College of Economic Sciences University, Baghdad, Iraq \\ ${ }^{2}$ Department of Computer Science, Baghdad College of Economic Sciences University, Baghdad, Iraq
}

\begin{abstract}
This work presents an approach for the applying Triple DES (TRIPLE DES) based on using genetic algorithm by adding intelligent feature for TRIPLE DES with $\mathrm{N}$ round for genetic algorithm. Encapsulated cipher file with special program which send an acknowledgment to a sender to know who decipher or broken to crash it, Thus it is considered as the initial step to improve privacy. The outcome for proposed system gives a good indication that it is a promising system compared with other type of cipher system.
\end{abstract}

Keywords: TRIPLE DES, Cryptography, privacy, security, round, encapsulation cipher file, Genetic algorithm.
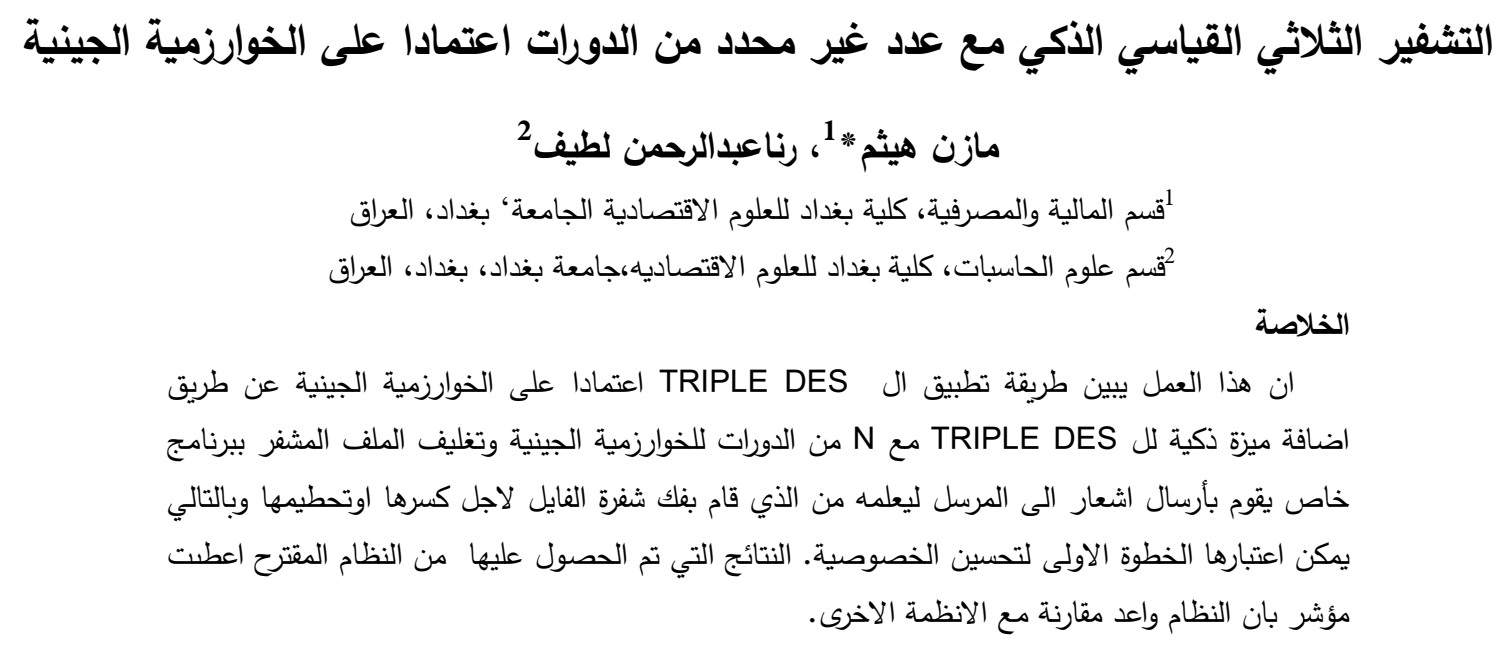

\section{Introduction}

The essential part of information technology is to achieve data security and privacy. Information security in data storage and transmission is becoming important as the fast grew of exchanging digital data in an electronic way. Cipher system considers to be the basic element for improving data security, in other hand decipher system is an important step for attack to broken secure data in all time, thus it must create a stamp for sender (cipher data) and one for who received(decipher) to guarantee the data received by a specific user [1]. Development of human intelligence with the art of cryptography has become more sophisticated in order to make information more secure. Cryptography using genetic algorithm has attracted more interest in recent years. There are primary types of cryptography a secret and a public key. Secret key cryptography is known as symmetric key cryptography where the encrypted (sender) and the decrypted (received) files has the same key. Public key cryptography is called asymmetric key cryptography which uses a pair of keys called private and public for encryption and decryption [2]. 
There are two ways of key production, the first one is mathematical like AES, DES and the other one is based on the theory of natural selection $[1,2]$.

Cryptography generally uses DES algorithm for the Encryption and Decryption. DES using round and round strategy. The DES uses private key and its works by using the same key to encrypt and decrypt a data [3].

Many genetic algorithms based encryption algorithms have been successfully used in many papers. The basic idea of research on GAs has been introduced in many researches which gives it a robustness in security confidence.

Jun Song,et. al., 2007[4], Their paper stated a way for using genetic algorithm in cryptanalysis of tworound DES .Depending on fitness function they adopted a known plaintext attack to produce a variety of optimum keys and count every bit of them one by one to find some valuable bits, which generate a significant deviation from the other bits, thus, the 56-bit key is successfully gained without searching the whole search space. An experimental result specified that this is a promising method and can works with the other complex block ciphers. Gove Nitinkumar Rajendra, et. al., 2011[5], they proposed a new method to data security based on brain mutually waves and genetic algorithm and with pseudorandom binary sequence for encrypt and decrypt the data. The properties of such a method comprise a high data security and high feasibility for practical application. Poornima Naik, et. al., 2014[6], in their paper they try to exploit the randomness in crossover and mutation processes for generating a pair of asymmetric key used for encrypt and decrypt a messages. In their work they have used four crossover points, three mutation points and a single random byte and a permutation factor. The use of randomness with permutation makes the algorithm more robust and hard to break. Suvajit Dutta, et. al., 2014[7], their paper deals with the confidentiality of electronic data which is transmitted over the internet by using the concept of genetic algorithms with pseudorandom function to encrypt and decrypt data stream. The encryption process is applied over a binary file. They proposed genetic algorithm depends on a method of encrypt a secret key which obviously it satisfied the goals that are required in any encryption method for encrypt binary files. Purvi Garg, et.al. 2015[8], in their paper they stated that ring crossover operator using genetic algorithms has been used in performing cryptanalysis of SDES. The scope of this paper is restricted to a cipher text attack. Keys are generated by different combinations using Genetic Algorithm and hence it is deduced that Genetic Algorithm is a better method than the Brute Force for analyzing SDES. Ms. B. D. Nagpure, et. al., ,2016[9],their paper stated at a cryptography based on Genetic Algorithm to implement security of information and data transmission so as to provide confidentiality, integrity, authentication and non-repudiation of the messages. A private key is used to encrypt a plain text of receiver to outcome an intermediate cipher which encrypted again using genetic algorithm to outcome a final cipher.

\section{Genetic algorithm}

The Genetic Algorithm (GAs) is a planning to move from one populace of "chromosomes" (or "bits") to another populace by utilizing a kind of "normal choice". Every chromosome comprises of "qualities" (e.g., bits), every quality being an example of a specific "allele" (e.g., 0 or 1). Hereditary calculations can be isolate into the accompanying three sorts of fundamental operation: selection, hybrid, and change. Selection depends on the wellness incentive to choose chromosomes in the populace for multiplication. The fitter the chromosome, the more circumstances it is probably going to be imitated. In Crossover a hybrid administrator has an essentialness as that of hybrid in a characteristic hereditary process. For instance, a strings 10000100 and 11111111 could be traversed after the third locus in each to deliver the two posterity 10011111 and 11100100 . The hybrid administrator generally imitates natural recombination between two single chromosome creatures [10]. In Mutation: it is a hereditary administrator arbitrarily flips a portion of the bits in a chromosome. For instance, the string 00000100 may be transformed in its second position to yield 01000100 . Transformation can happen at each piece position in a string with some likelihood, typically little. [11, 12]

\section{TRIPLE DES}

TRIPLE DES or the Triple Data Encryption Algorithm (TDEA) was produced to address the conspicuous blemishes in DES without outlining a radical new cryptosystem. It additionally has the benefit of demonstrated unwavering quality and a more extended key length that takes out a large number of the assaults that can be utilized to lessen the measure of time it takes to break DES [13]. Information Encryption Standard (DES) utilizes is a 56-bit key and isn't considered appropriate to 
encode oversensitive information. TRIPLEDES essentially broadens the key size of DES by execute the calculation three times in progression utilizing three different keys. The consolidated key size is in this manner 168 bits (3 times 56). TDEA includes with three 64-bit DEA keys (K1, K2, K3) in the mode Encrypt-Decrypt-Encrypt (EDE), that is, the plain content is scrambled with K1, at that point unscrambled with K2, and afterward encoded with K3 [14]. The guidelines represents three of keying choices:

1 -The more favored alternative, actualizes three commonly free keys $(\mathrm{K} 1 \neq \mathrm{K} 2 \neq \mathrm{K} 3 \neq \mathrm{K} 1)$. It gives key space of $3 \times 56=168$ bits.

2-Implement two commonly autonomous keys and a third key that is the same as the main key $(\mathrm{K} 1 \neq$ $\mathrm{K} 2$ and $\mathrm{K} 3=\mathrm{K} 1)$. This gives key space of $2 \times 56=112$ bits.

3-a key heap of three comparable keys $(\mathrm{K} 1=\mathrm{K} 2=\mathrm{K} 3)$. This choice is comparable to DES Algorithm. In TRIPLEDES the three times emphasis is connected to build the encryption level and normal time $[15,16]$. Triple DES runs three times slower than DES, however is considerably more secure and confident if utilized appropriately [17].

\section{Proposed system:}

The information transferring through e-environment thus must improve the data privacy and security between sender and receiver to avoid any intrusion or damage on transfer data. In this section a new feature to TRIPLE DES add by merge it with genetic Algorithm and then covered with any executable file with track ability.

The first step to re-code TRIPLE DES file is start with genetic algorithm thus dealing with data that based on ASCII.

Note ASCII code start from 0 to 255 in binary system $=2^{8}$ refer to 8 bit for all character of input file as shown in Table-1.

In this work the following algorithms have been used to implement the proposed system:

\section{Algorithm 1}

Main algorithm

Input (TRIPLE DES file)

Output (cipher file)

1- initial population from plan-text(TRIPLE DES output) (by div block as chromosomes )

2- Genetic sub

2.1 calculate fitness function (depend on privilege )

2.2 genetic operation

2.3 save data

2.4 goto 2.1

3- Detect sender TX and receiver RX

4- truck it

5- if attack occurring then save in log file TX /RX (depend on acknowledge )

6- encapsulation function

7- goto 2 increment privilege

\section{Algorithm 2}

fitness function

$1 *$

depend on privilege

privilege mean as the following example

if current string

$$
\text { Line } 1=\underline{A} \text { B C D E F G H }
$$

as input for TRIPLE DES and output of TRIPLE DES as:

Line 2=

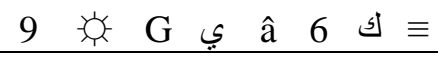

And ASCII cod

\begin{tabular}{|l|l|l|l|l|l|l|l|l|}
\hline 57 & 15 & 71 & 239 & 131 & 231 & 54 & 232 & 240 \\
\hline
\end{tabular}

Then binary convert as

\begin{tabular}{|l|l|l|l|l|l|l|}
00111001 & 00001111 & 10000011 & 11100111 & 00110110 & 11101000 & 11110000
\end{tabular}

Check if random number add to current ASCII is not near to source data and not same number.

example

code cod 
$\mathrm{A} \rightarrow 65 \rightarrow 57 \rightarrow 9$

Which refer to random increased code

*/Input( process TRIPLE DES line of 8 character )

Output( new line)

1 -start

2-if call function(sum_of_random) $>3$ then line_ok $=$ true

3-end

\section{Algorithm 3}

function(sum_of_random) as integer

input (line1, line 2)

output (integer value of randomity)

1- start

2- loop

check 16ine 1[i], line 2[i] different

if different $>3$ and $i<6$ then exit

$$
\begin{gathered}
\text { i+++ } \\
\text { until } i>8
\end{gathered}
$$

3- end

\section{algorithm 4}

// track algorithm

Input (cipher file )

Output(cipher with sender and receiver rout [primary and secondary], flag )

1- start

2- read cipher file and detect sender

3- read receiver by detect Primary rout and secondary rout )

4- send file and check

5 - if same rout then flag $=$ true else flag $=$ false

6- end

\section{algorithm 5}

// same rout (using to track)

Input (sender and receiver rout [primary and secondary], acknowledge)

Output(true |false)

1- start

2- sum++

3- read current station

4- if current station in( primary or secondary)station list then sum --

5 - if sum $=0$ then

output=true

else

output=false

end if

6- end

\section{algorithm 6}

// encapsulation output

Input (cipher data)

Output(encapsulation cipher data)

1- start

2- select encapsulation method with (exe format, JPG format .....etc.)

3- implementation of selection method on input

4- end

\section{Experimental result:}

Using a simple file of TRIPLE DES as a segment test to implement it is shown in Figure-1 and Figure-2. The complexity test depends on irregular value that come and give final cipher file as shown in a curve in Figure-3and Figure-4 represent the complexity chart result from Table-2. 


\section{Conclusion}

1-cipher /decipher time is high speed when comparing current algorithm with another cipher algorithm using genetic as main engine.

2- new feature for algorithm add with detect whom receive.

3 -while increasing cipher file size then increase complexity because of need more time to analysis file. 4-security while any problem appear with file automatic acknowledgement will be send to sender(as new function).

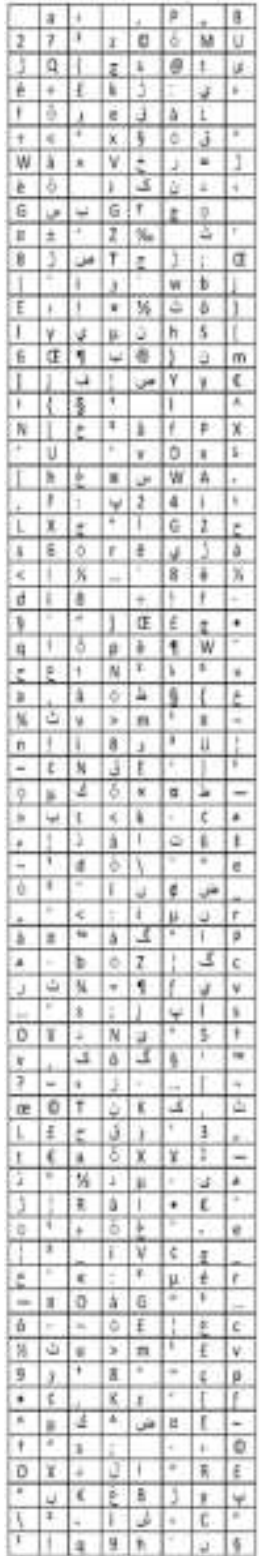

IIIDES block

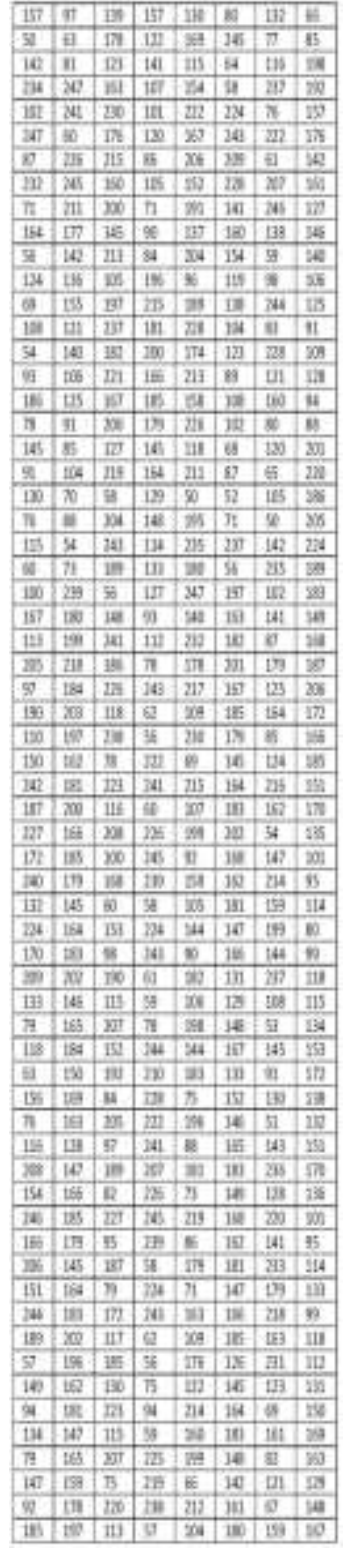

\begin{tabular}{|l|l|l|l|l|l|l|l|}
\hline 10041101 & 01100001 & 10001011 & 1001110 & 10000010 & 01010000 & 10000100 & 01000010 \\
\hline
\end{tabular}

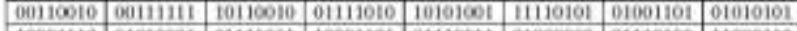

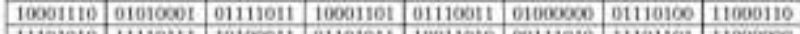

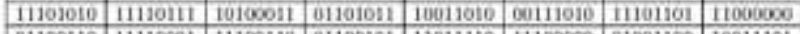

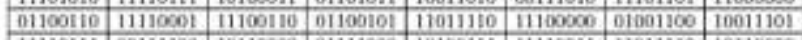

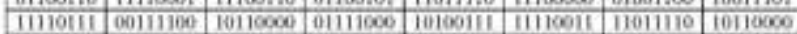

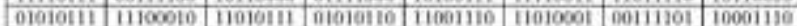

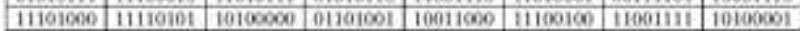

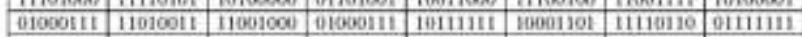

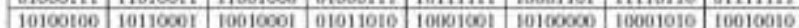

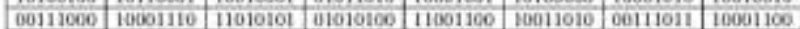

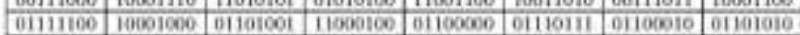

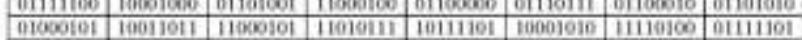

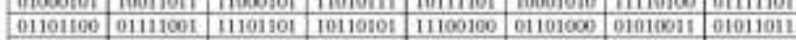

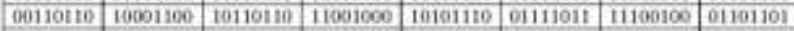

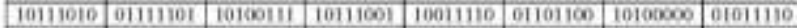

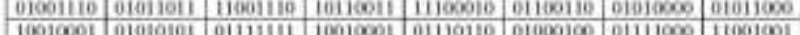

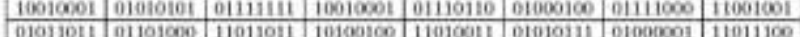

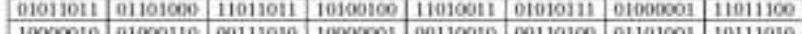

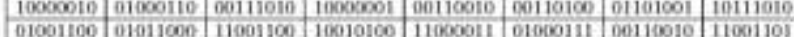
01110011 00110110 11110011 01110010 11101011 11101301 10001110 11100000

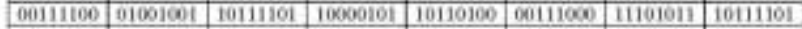
\begin{tabular}{|l|l|l|l|l|l|l|l|}
\hline Bi100000 & 11101111 & n60111000 & 01111111 & 11110111 & 11050101 & 0.100110 & 10110111 \\
\hline
\end{tabular}

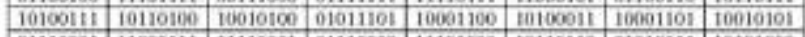

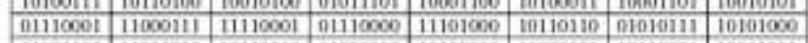

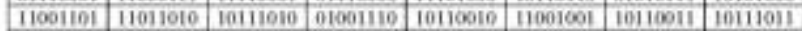

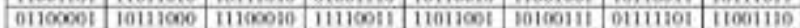

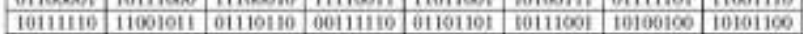

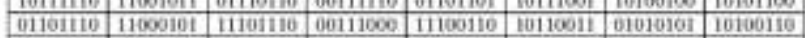

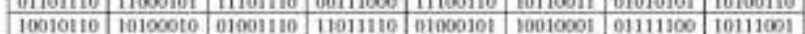
\begin{tabular}{|l|l|l|l|l|l|l|l|l|}
\hline 1110010 & 10110101 & 11011111 & 11110001 & 11010311 & 10100100 & 11011000 & 10010111 \\
\hline
\end{tabular} \begin{tabular}{|l|l|l|l|l|l|l|l|}
\hline 10111011 & 1100100 & 01110100 & no111100 & 01101011 & 10110111 & 10100010 & 10101010 \\
\hline
\end{tabular}

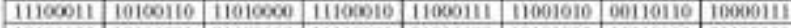

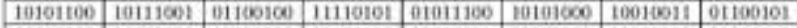

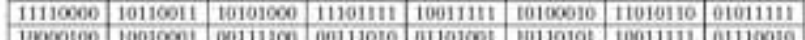

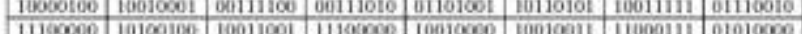

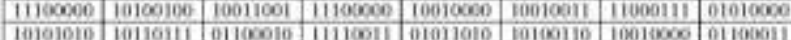

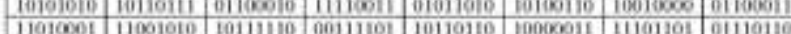

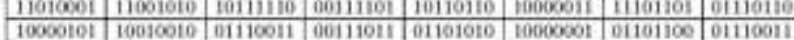

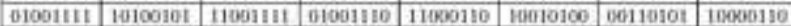
\begin{tabular}{|l|l|l|l|l|l|l|l}
\hline 01110110 & 10111000 & 10011000 & 11110100 & 10010000 & 6010011 & 10010001 & 10011001 \\
\hline
\end{tabular}

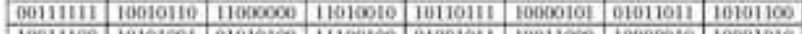

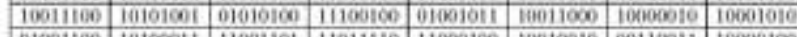

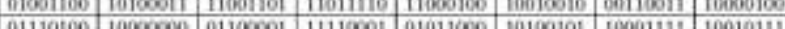

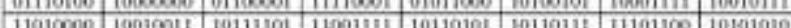

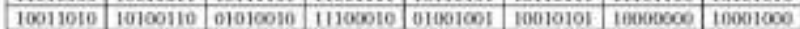

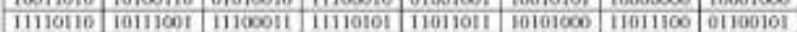

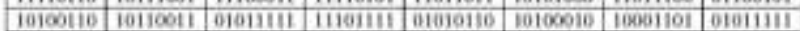
\begin{tabular}{|l|l|l|l|l|l|l|l|}
\hline 1100110 & 10010001 & L 0111011 & 0011010 & 10110011 & 10110105 & 11101001 & 01110010 \\
\hline
\end{tabular}

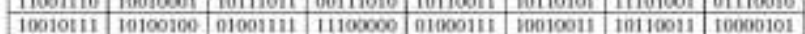
\begin{tabular}{|l|l|l|l|l|l|l|l|}
\hline 11110160 & 10110111 & 10101100 & 11110011 & 10100011 & 10100110 & 11011010 & 01100011 \\
\hline
\end{tabular}

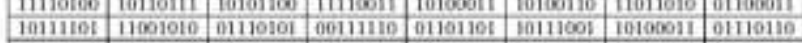
\begin{tabular}{|l|l|l|l|l|l|l|l|}
\hline 00111001 & 11000100 & 10111001 & 00111000 & 10110000 & 01111110 & 11100111 & 01110000 \\
\hline
\end{tabular} \begin{tabular}{|c|c|c|c|c|c|c|c|}
\hline 10010101 & $101000 t 0$ & 10060010 & 01001011 & 01111010 & 10010001 & 01111011 & 10006011 \\
\hline
\end{tabular}

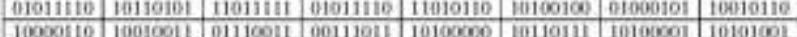

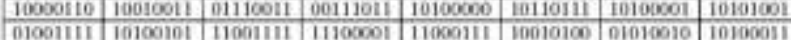

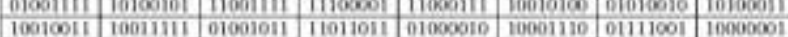
\begin{tabular}{|l|l|l|l|l|l|l|l|}
\hline 01011100 & 10110010 & 11011100 & 11101110 & 11010100 & 10100001 & 01000011 & 10010100 \\
\hline
\end{tabular}

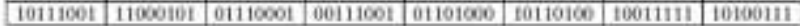

Figure 1-initial input of 64 line of 8 character to process

(b)

(c)

ASCII code for input convert input ASCII to binary 


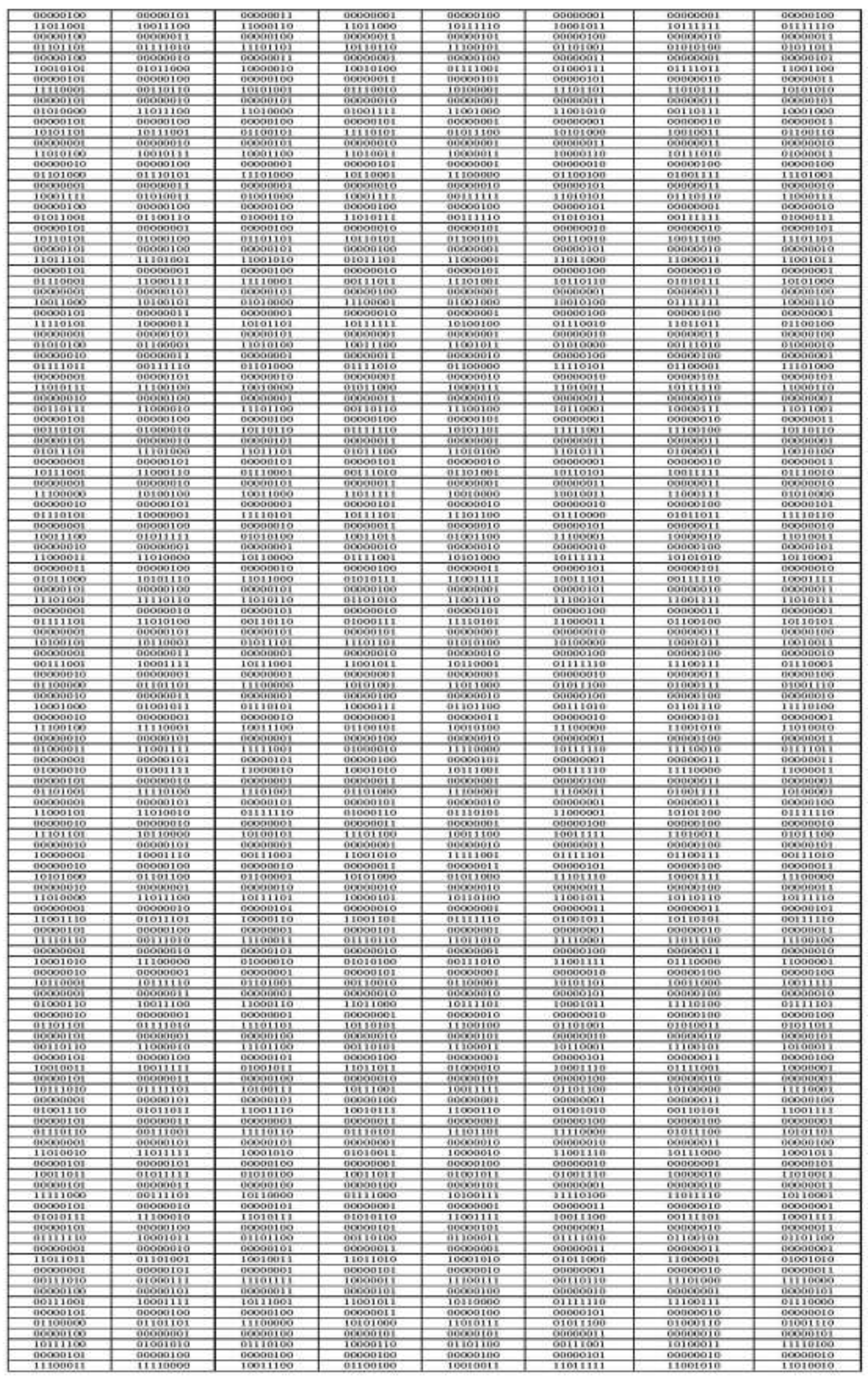

Figure 2-process result for TRIPLE DES 
Table 1-

\begin{tabular}{|c|c|c|}
\hline III DES & Cipher & Different \\
\hline 157 & 160 & 3 \\
\hline 97 & 101 & 4 \\
\hline 139 & 141 & 2 \\
\hline 157 & 161 & 4 \\
\hline 130 & 132 & 2 \\
\hline 80 & 85 & 5 \\
\hline 132 & 137 & 5 \\
\hline 66 & 68 & 2 \\
\hline 50 & 52 & 2 \\
\hline 63 & 64 & 1 \\
\hline 178 & 181 & 3 \\
\hline 122 & 124 & 2 \\
\hline 169 & 172 & 3 \\
\hline 245 & 248 & 3 \\
\hline 77 & 79 & 2 \\
\hline 85 & 88 & 3 \\
\hline 142 & 146 & 4 \\
\hline 81 & 82 & 1 \\
\hline 123 & 126 & 3 \\
\hline 141 & 142 & 1 \\
\hline 115 & 119 & 4 \\
\hline 64 & 67 & 3 \\
\hline 116 & 117 & 1 \\
\hline 198 & 203 & 5 \\
\hline 234 & 239 & 5 \\
\hline 247 & 251 & 4 \\
\hline 163 & 167 & 4 \\
\hline 107 & 110 & 3 \\
\hline 154 & 159 & 5 \\
\hline 58 & 59 & 1 \\
\hline 237 & 240 & 3 \\
\hline 192 & 195 & 3 \\
\hline 102 & 107 & 5 \\
\hline 241 & 243 & 2 \\
\hline 230 & 235 & 5 \\
\hline 101 & 104 & 3 \\
\hline 222 & 223 & 1 \\
\hline 224 & 228 & 4 \\
\hline 76 & 79 & 3 \\
\hline 157 & 158 & 1 \\
\hline 247 & 249 & 2 \\
\hline 60 & 61 & 1 \\
\hline 176 & 177 & 1 \\
\hline 120 & 122 & 2 \\
\hline 167 & 170 & 3 \\
\hline 243 & 245 & 2 \\
\hline 222 & 226 & 4 \\
\hline 176 & 181 & 5 \\
\hline 87 & 90 & 3 \\
\hline 226 & 230 & 4 \\
\hline 215 & 217 & 2 \\
\hline 86 & 90 & 4 \\
\hline 206 & 209 & 3 \\
\hline 209 & 214 & 5 \\
\hline 61 & 66 & 5 \\
\hline 142 & 146 & 4 \\
\hline 232 & 237 & 5 \\
\hline 245 & 248 & 3 \\
\hline 160 & 164 & 4 \\
\hline 105 & 108 & 3 \\
\hline 152 & 157 & 5 \\
\hline 228 & 233 & 5 \\
\hline 207 & 209 & 2 \\
\hline 161 & 164 & 3 \\
\hline
\end{tabular}




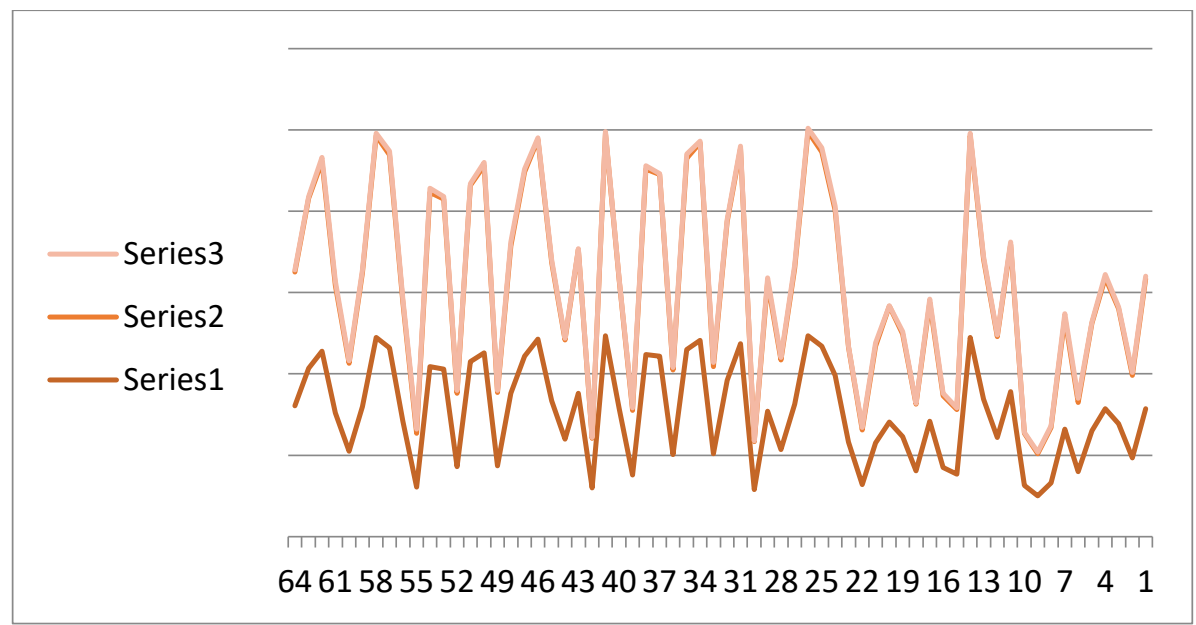

Figure 3-complexity chart for irregular value

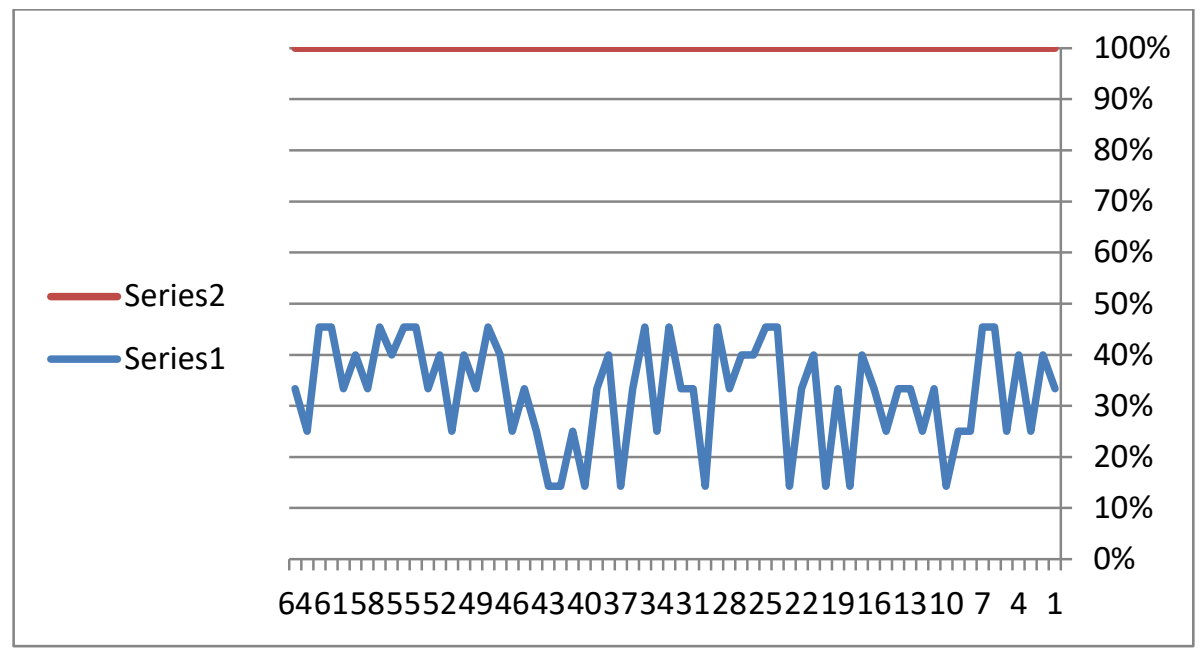

Figure 4-complexity chart for different value

Table 2- ASCII code for character and its binary representation

\begin{tabular}{|c|c|c|}
\hline ASCII & char & Binary \\
\hline 65 & A & 01000001 \\
\hline 66 & B & 01000010 \\
\hline 89 & Y & 01011001 \\
\hline 90 & Z & 01011010 \\
\hline
\end{tabular}

\section{References}

1. Jhingran, R., Thada, V. and Dhaka, S. 2015. A Study on Cryptography using Genetic Algorithm", International Journal of Computer Applications, 118(20): 10 - 14.

2. Goyat, S. 2012. Cryptography Using Genetic Algorithms. IOSR Journal of Computer Engineering (IOSRJCE), 1(5): 06-08.

3. Nagpure, B. D. , Dhote, A. D., Rokade P. S., Kale P. B. and Kinhikar, N. S. 2016. Implementation of Network Security Using Genetic Algorithm, International Journal of Research in Advent Technology (IJRAT) ,Special Issue ,National Conference "Convergence 2016", 06th-07th April.

4. Song, J., Zhang, H., Meng, Q. and Wang, Z. 2007. Cryptanalysis of Two-Round DES Using Genetic Algorithms", Springer-Verlag Berlin Heidelberg, pp: 583-590.

5. Rajendra, G. N. and kaur, B. R. 2011. A New Approach for Data Encryption Using Genetic Algorithms and Brain Mu Waves", International Journal of Scientific and Engineering Research. 2(5): 01-04. 
6. Naik, P. and Naik, G. 2014. Asymmetric Key Encryption using Genetic algorithm, International Journal of Latest Trend in Engineering and Technology, (IJLTET), 3(3).

7. Dutta S., Das T., Jash S., Patra D. and Paul P. 2014. A Cryptography Algorithm Using the Operations of Genetic Algorithm \& Pseudo Random Sequence Generating Functions, International Journal of Advances in Computer Science and Technology, 3(5): 325-330.

8. Garg P. and Bhardwaj, S. 2015. Cryptanalysis of Simplified Data Encryption Standard Using Genetic Algorithm. American Journal of Networks and Communications. 4(3): 32-36.

9. Nagpure B. D., Dhote A. D., Rokade P. S., Kale P. B. and Kinhikar N. S . 2016. Implementation of Network Security Using Genetic Algorithm, International Journal of Research in Advent Technology (IJRAT) (Special Issue) National Conference "CONVERGENCE 2016", 06th-07th April.

10. Bhasin H. and Bhatia S. 2011. Application of Genetic Algorithms in Machine learning”, IJCSIT, 2(5).

11. Mitchell Melanie.1999. An Introduction to Genetic algorithm, A Bradford Book, The MIT Press.

12. Almarimi A. 2010. A New Approach For Data Encryption Using Genetic Algorithms, and Published in: - Proceeding CERMA '10 Proceedings of the IEEE Electronics, Robotics and Automotive Mechanics Conference.

13. Triple Data Encryption Standard (Triple-DES), ttps://www.vocal.com/cryptography/tdes/ Triple Data Encryption Standard (Triple-DES).html.

14. "3DES", http://www.cryptosys.net/3des.html.

15. Kakkar A, Singh M. L. and Bansal P.K. 2012. Comparison of Various Encryption Algorithms and Techniques for Secured Data Communication in Multinode Network, International Journal of Engineering and Technology, 2(1): 87-92.

16. Kumar A., Jakhar S. and Makkar S. 2012. Comparative Analysis between DES and RSA Algorithm's, International Journal of Advanced Research in Computer Science and Software Engineering, 2(7): 386-391.

17. Aamer, N. 2005. "A Performance Comparison of Data Encryption Algorithm," IEEE. 